\title{
Algoritmo de Posicionamento de Regeneradores e Comprimentos de onda em Redes Ópticas
}

\author{
Rodrigo, F. S. C. Oliveira \\ Escola Politécnica de Pernambuco \\ Universidade de Pernambuco \\ 50.720-001 - Recife, Brasil \\ rodrigofsc@ hotmail.com
}

\author{
Carmelo J. A. Bastos-Filho \\ Escola Politécnica de Pernambuco \\ Universidade de Pernambuco \\ 50.720-001 - Recife, Brasil \\ carmelofilho@ecomp.poli.br
}

Resumo Neste artigo nós propomos um algoritmo de otimização multiobjetiva evolucionaria para posicionamento de regeneradores e comprimento de onda em redes ópticas. Visando realizar o posicionamento de regeneradores nos nós e o posicionamento de comprimentos de onda nos enlaces considerando o desempenho(probabilidade de bloqueio) e o custo da rede. O algoritmo multiobjetivo evolucionário utilizado no processo de otimização é baseado no SPEAII que está descrito em [11].

\footnotetext{
Abstract In this paper we propose the use a multiobjective evolutionary optimization algorithm for regenerator and wavelength placements in optical network. The aim is to perform regenerator placement in the nodes and wavelength placement in the links considering network performance (blocking probability) and capital cost. The multi-objective evolutionary algorithm used in the optimization process is the SPEA2 that is described in [11].
} 


\section{Introdução}

Redes ópticas translucidas são alternativas de desempenho e custo eficazes para redes transparentes e opacas[3,4]. Nessas redes alguns nós são transparentes e outros têm capacidades de regeneração. Duas estratégias são possíveis para projetar redes translucidas: Ilhas de Transparência [5] e Sparse Regeneration [3,5]. Nesse artigo foi utilizada a abordagem Sparse Regeneration para projetar redes ópticas translucidas. Duas questões naturalmente nascem nesse cenário: quais nós posicionar regeneradores e como usar a esses regeneradores de acordo com a demanda em redes com trafego dinâmico. $\mathrm{O}$ algoritmo responsável pela escolha do numero de regenerados e em que nós eles devem ser posicionados é conhecido como algoritmo de posicionamento de Regeneradores (RP). O algoritmo que decide em que nó de uma dada rota o sinal óptico deve ser regenerado é conhecido como algoritmo de alocação de regenerador (RA)[3].

Algoritmos Multiobjetivos Evolucionários vêm sendo utilizados para resolver problemas que ocorrem em redes ópticas como descrito em $[6,7,8]$. Em sua grande maioria eles apresentam excelentes resultados.

Nesse artigo, é apresentado um algoritmo para posicionamento de regeneradores e comprimentos de onda. Esse artigo propõe o uso de um MOEA, nesse caso SPEAII, para resolver o problema de posicionamento de regeneradores e comprimentos de onda, aqui chamado de Algoritmo Multiobjetivo evolucionário posicionamento de regeneradores e comprimentos de onda (MORWP). $\mathrm{O}$ algoritmo considera três objetivos: a probabilidade de bloqueio como variável de desempenho, o custo da rede e o numero de nós translúcidos como variáveis econômicas. A justificativa do numero de nós translucido ser considerado uma variável econômica será demonstrado mais a frente.

O sinal óptico será regenerado usando elementos da rede que são capazes de executar a regeneração $3 \mathrm{R}$ (reamplifying, re-shaping and re-timing) $\mathrm{O} / \mathrm{E} / \mathrm{O}$. O algoritmo de posicionamento de regeneradores e comprimentos de onda (RWP) avalia a probabilidade de bloqueio usando um modelo de camada física que considera as seguintes penalidades: ruído ASE, efeito de saturação de ganho do amplificador, saturação de ruído ASE em EDFAs, crosstalk em switches ópticos, dispersão cromática residual, mistura de quatro ondas (FWM) e efeitos PMD em fibras de transmissão. Esse modelo de camada física está descrito em [10]. Esse é o primeiro artigo do nosso conhecimento que propõe uma solução para o problema (RWP).

\section{Descrição e Representação do Pro- blema}

\subsection{Problema de Posicionamento de Regene- radores e Comprimentos de onda}

Nessa seção será descrita a formulação adotada para o RWP e como a rede e seus recursos são representados. Portanto, esse artigo se concentra no seguinte problema: dada uma determinada topologia de rede com os algoritmos de roteamento e atribuição de comprimento de onda estabelecidos, é preciso determinar quais nós da rede devem ter a capacidade de regeneração e quantos comprimentos de onda tem em cada enlace tentando minimizar o custo e maximizar o desempenho da rede, simultaneamente. Para avaliar o desempenho da rede usamos simulações para obter a probabilidade de bloqueio. Propomos dois modelos de custo para considerar o custo da rede como uma variável econômica nesse problema. O primeiro é o custo da rede e o segundo é relacionado com o custo do enlace. Para avaliar o custo total da rede para cada solução adicionasse esses dois modelos de custo.

\subsection{Representação do Posicionamento de Re- generadores e Comprimentos de Onda}

O primeiro passo para construir um algoritmo de posicionamento de regeneradores e comprimentos de onda é determinar uma representação apropriada para localização dos nós translúcidos e o numero de regeneradores posicionados nesses nós. O segundo passo é como representar os enlaces e o numero de comprimentos de onda disponível em cada enlace. Isso é possível através da representação dessas características com um vetor de inteiros. Então definimos o vetor $V=\left\{v_{i}\right\}, i \in 1,2,3, \ldots, N, N+1, \ldots, L$ onde $N$ é o numero de nós na rede e $L-N$ é o numero de enlaces na rede, $\log o L$ é o numero de nós mais o numero de enlaces. Portanto, para cada inteiro $i \leq N, v_{i}$ é o numero de regeneradores implantados no nó $i$ e para cada inteiro $N+1 \leq i \leq L, v_{i}$ é o numero de comprimentos de onda no enlace $i$.

\subsection{Modelo de Custo}

Para execução do MORWP um modelo de custo foi proposto. Esse modelo é composto de duas variáveis econômicas: O custo do nó e o custo do enlace. No primeiro, é considerado o numero de regeneradores implantados nos nós translúcidos e características dos OXC. No segundo, o numero de comprimentos de onda em cada enlace. O modelo de custo utilizado está descrito abaixo: 


$$
\begin{gathered}
C_{\text {total }}=C_{\text {Nó }}+C_{\text {Enlace }} \\
C_{N o ́}=1.4 R+C_{\text {OXC }} \\
C_{\text {OXC }}=[(0.0522 \mathrm{~W}+6.24) G+2.5] \\
C_{\text {Enlace }}=1.0 \mathrm{~W}
\end{gathered}
$$

Onde $R$ é o numero de pares de regeneradores posicionados no nó. $W$ é o numero de pares de comprimentos de ondas no enlace. Por fim, $G$ é o grau do nó.

\section{Algoritmo Multiobjetivo evolucio- nário de Posicionamento de Rege- neradores e Comprimentos de Onda}

Nessa seção será descrito o algoritmo de otimização multiobjetiva evolucionaria usada na distribuição de regeneradores e comprimentos de onda tentando minimizar o custo e a probabilidade de bloqueio da rede simultaneamente. A probabilidade de bloqueio é uma medida relativa de quantas chamadas são bloqueadas pela rede devido à falta de recursos físicos ou porque o lightpath não atingiu o QoS exigido. No entanto, é um importante indicador de qualidade da rede. O custo da rede é importante no processo de RWP, pois podemos chegar a uma rede de alta qualidade com um pequeno custo.

Algoritmos evolucionários vêm se mostrando eficientes soluções para problemas multiobjetivos. Para execução da otimização multiobjetiva, foi usado o algoritmo multiobjetivo evolucionário (MOEA) chamado SPEAII. O SPEAII foi proposto por Zitzler ET AL [11] e foi baseado em algoritmos genéticos (GA)[12]. O pseudocódigo do SPEAII pode ser consultado em [11]. A principal diferença entre o SPEAII e um GA qualquer é principalmente a forma como eles praticam o elitismo através do operador de seleção natural, a forma como ocorre a atribuição do fitness e o uso de um arquivo externo. Todas essas diferenças são explicadas em [13].

Em algoritmos genéticos, cada individuo representa uma possível solução para o problema. $\mathrm{O}$ individuo é representado pelo vetor $V$ definido na seção anterior. Os indivíduos são formados por genes que são as variáveis de decisão do problema e são representados por cada valor $v_{i}$. Os genes nesse problema foram tratados de maneira diferente. Para cada $i \leq n$ valor , representando o numero de regeneradores nos nós, o valor máximo de $v_{i}$ é 200 e o valor mínimo é 0 , no caso em que o nó não é translucido. Mas para cada $N+1 \leq i \leq L$, representando os comprimentos de ondas disponíveis nos enlaces, o máximo valo de é 40e o mínimo é 2 . A granularidade do crescimento ou decrescimento do valor de cada gene é decrescente ao longo das gerações. Começam em 8 passando por 4 após 50 gerações e chegando a 2 após outras 50 gerações. Ao chegar ao valor de 2 a granularidade permanece constante durante o resto das iterações.

Em um GA genérico, a qualidade do individuo é determinado pelo fitness. Nos MOEA a qualidade do individuo está relacionado ao fitness que é obtido através da avaliação de todos os objetivos e o critério de dominância entre os indivíduos da população. No RWP avaliamos três objetivos. O primeiro é o custo total da rede representada pelo individuo. O segundo é o desempenho da rede através da probabilidade de bloqueio, usando os resultados de simulações no simulador que implementa o modelo de camada física. Por fim, o terceiro é o número de nós translúcidos que consideramos uma variável econômica. Os numero de nos translúcidos é considerado nesse artigo como uma variável econômica, pois ela influi diretamente no custo de implantação e no custo de manutenção da rede. Quanto menor o número de nós translúcidos menor o custo de implantação pois não será necessário algumas estruturas para a implantação dos regeneradores. Por outro lado, quanto menor o numero de nós translúcidos menor será o custo de manutenção, pois serão menos nós para fazer a manutenção dos regeneradores.

Durante cada iteração, operadores genéticos são usados pelo GA: Crossover, Mutação e Seleção. A seleção é executada em duas fases. A primeira é a seleção do ambiente que seleciona os melhores indivíduos do arquivo externo em termos de fitness, mas preservando a diversidade entre as soluções. Então o algoritmo executa o torneio binário nesses indivíduos selecionados para o arquivo externo obtendo pares de indivíduos escolhidos aleatoriamente. Esses pares de indivíduos obtidos na seleção sofrem Crossover e mutação para formar a próxima população. O pseudocódigo de cada rotina está descrita em [11]. O operador de Crossover utilizado nesse algoritmo foi o Crossover de multiponto com probabilidade de Crossver $P_{c}$. O operador de Mutação utilizado foi a mutação per gene uniforme onde o gene escolhido para sofrer mutação é substituído por um valor aleatório entre seus limites. A mutação tem uma probabilidade de mutação $P_{m}$. O algoritmo proposto nesse artigo está descrito no algoritmo 1 . 


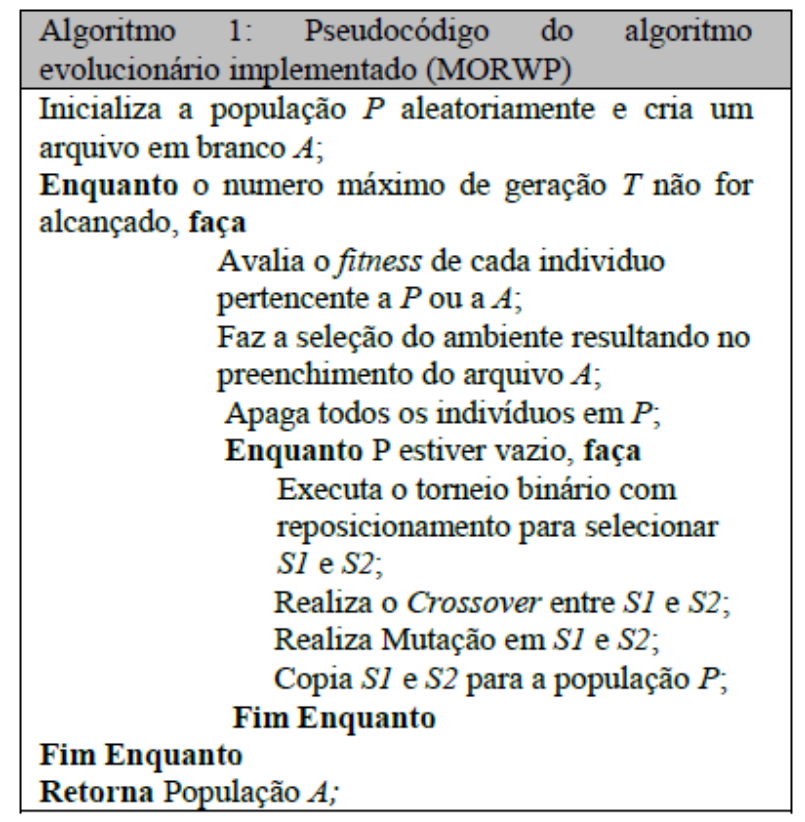

O algoritmo MORWP desenvolvido nesse artigo recebe três parâmetros como entrada: $\mathrm{O}$ numero de indivíduos no arquivo externo $A$, o numero de indivíduos na população $P$ e o numero de gerações $T$ que ele tem que evoluir. Por fim, $\mathrm{O}$ algoritmo retorna a população no arquivo externo $A$.

\section{Resultados de Simulações}

Os resultados obtidos mostrados nesse artigo foram tirados a partir de simulações. A Topologia de rede Pacific Bell Modificada mostrada na figura 1 foi utilizada nas simulações e na tabela 1 estão os parâmetros de simulação

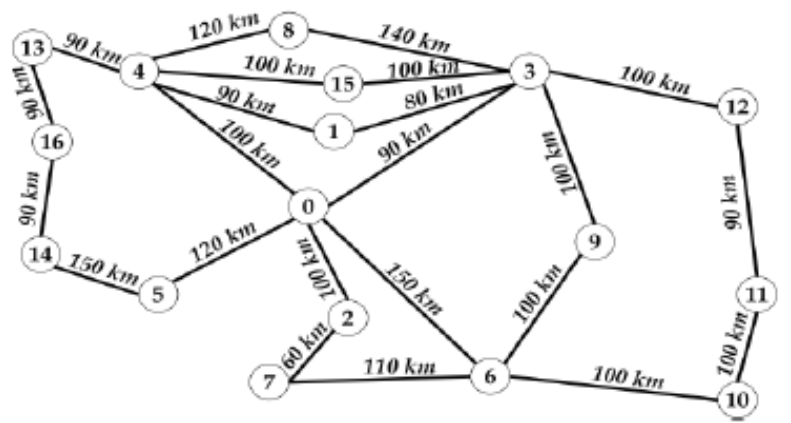

Figura 1 - Topologia Pacific Bell Modificada.
Tabela 1 - Parâmetros de Simulação

\begin{tabular}{|c|c|c|}
\hline Parâmetro & Valor & Definição \\
\hline Psat & $20 \mathrm{dBm}$ & $\begin{array}{l}\text { Potência de Saturação na saída } \\
\text { do amplificador. }\end{array}$ \\
\hline$O S N R_{\text {in }}$ & $40 \mathrm{~dB}$ & $\begin{array}{l}\text { Relação sinal-rúdo óptica na } \\
\text { entrada. }\end{array}$ \\
\hline OSNR & $20 \mathrm{~dB}$ & $\begin{array}{l}\text { Relação sinal-rúdo óptica } \\
\text { adotada como critério de QoS. }\end{array}$ \\
\hline$B$ & 40 Gbp5 & Taxa de transmissão. \\
\hline$B_{o}$ & $100 \mathrm{Ghz}$ & $\begin{array}{l}\text { Largura de banda a } 3 \mathrm{~dB} \text { do } \\
\text { filtro óptico. }\end{array}$ \\
\hline$\Delta f$ & $100 \mathrm{Ghz}$ & $\begin{array}{l}\text { Espaçamento de frequência } \\
\text { entre canais. }\end{array}$ \\
\hline$\lambda_{i}$ & $\begin{array}{l}1528.77 \\
\mathrm{~nm}\end{array}$ & $\begin{array}{l}\text { Menor comprimento de onda } \\
\text { pertencente a grade. }\end{array}$ \\
\hline$\lambda_{0}$ & $1557 \mathrm{~mm}$ & $\begin{array}{l}\text { Comprimento de onda cujo } \\
\text { dispersão é igual a } 0 .\end{array}$ \\
\hline$\lambda_{\text {OED }}$ & $\begin{array}{l}1544.53 \\
\operatorname{mm}\end{array}$ & $\begin{array}{l}\text { Comprimento de onda cujo } \\
\text { dispersão residual é igual a } 0 \text {. }\end{array}$ \\
\hline$\alpha$ & $\begin{array}{l}0.2 \\
\mathrm{~dB} / \mathrm{Km} \\
\end{array}$ & $\begin{array}{l}\text { Coeficiente de atenuação na } \\
\text { fibra. } \\
\end{array}$ \\
\hline$L_{\text {Min }}$ & $3 \mathrm{~dB}$ & Perda do multiplexador. \\
\hline$L_{\text {Domer }}$ & $3 \mathrm{~dB}$ & Perda do demultiplexador \\
\hline$L_{\text {Simitach }}$ & $3 \mathrm{~dB}$ & Perda do switch. \\
\hline$F_{0}$ & $6 \mathrm{~dB}$ & $\begin{array}{l}\text { Fator de ruido do amplificador } \\
\text { para baixos valores de } \\
\text { potências. }\end{array}$ \\
\hline$\varepsilon$ & $-40 \mathrm{~dB}$ & Fator de isolação do switch. \\
\hline$D_{P M D}$ & $\begin{array}{l}0.05 \\
\mathrm{ps} / \mathrm{km}^{1 / 2}\end{array}$ & Coeficiente de PMD. \\
\hline$S_{D C F}$ & \begin{tabular}{|l}
1.87 \\
$\mathrm{ps} / \mathrm{kmn} \mathrm{n}$ \\
$\mathrm{m}^{2}$ \\
\end{tabular} & $\begin{array}{l}\text { Coeficiente da fibra } \\
\text { compensadora de dispersão. }\end{array}$ \\
\hline$S_{7 x}$ & $\begin{array}{l}0.06 \\
\mathrm{ps} / \mathrm{kmn} \mathrm{n} \\
\mathrm{m}^{2}\end{array}$ & $\begin{array}{l}\text { Coeficiente de dispersão na } \\
\text { fibra de transmissão. }\end{array}$ \\
\hline $\begin{array}{l}\text { Carga da } \\
\text { Rede }\end{array}$ & $120 \mathrm{Erl}$ & Carga da rede. \\
\hline
\end{tabular}

Para execução do MORWP foram selecionados três cenários de destaques. Eles possuem muitos dos parâmetros iguais. Possuem tamanho do arquivo e população igual a 75. Usam o operador de crossover de multiponto com taxa de $90 \%$, limitam as variáveis de 0 à 200 para os regeneradores e de 0 à 40 para os comprimentos de onda. Suas granularidades são variáveis e começam em 8 e a cada 50 gerações decaem de $50 \%$ terminando em 2. Suas diferenças estão explicitadas na tabela 2 . 
Tabela 2 - Descrição dos cenários de simulação utilizados.

\begin{tabular}{|l|c|c|c|}
\hline Cenário & Crossover & Mutação & $\begin{array}{c}\text { Métodos } \\
\text { adaptativos }\end{array}$ \\
\hline B & $\begin{array}{c}0,9 \\
\text { MultiPonto }\end{array}$ & $\begin{array}{c}0,1 \\
\text { Uniforme }\end{array}$ & $\begin{array}{c}\text { Probabilidade } \\
\text { de Bloqueio }\end{array}$ \\
\hline C & $\begin{array}{c}0,9 \\
\text { MultiPonto }\end{array}$ & $\begin{array}{c}0,1 \\
\text { Discreta }\end{array}$ & Mais Usado \\
\hline D & $\begin{array}{c}0,9 \\
\text { MultiPonto }\end{array}$ & $\begin{array}{c}0,1 \\
\text { Uniforme }\end{array}$ & Mais Usado \\
\hline
\end{tabular}

Para melhor argumentação não será mostrado os paretos em três dimensões já que são de difícil percepção. Os gráficos das figuras 2, 3, 4 são gráficos que mostram o custo e a probabilidade de bloqueio de cada solução dos cenários escolhidos e detalhados na tabela 2. Essas soluções também foram escolhidas para analise mais detalhada e são as soluções que serão comparadas com as soluções de algoritmos de RP presentes na literatura.

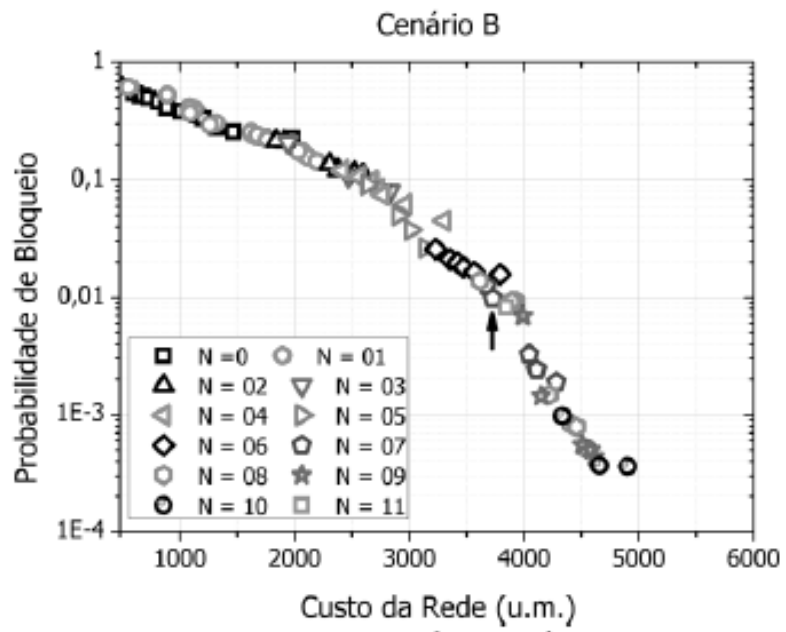

Figura 2 - Pareto do cenário B.

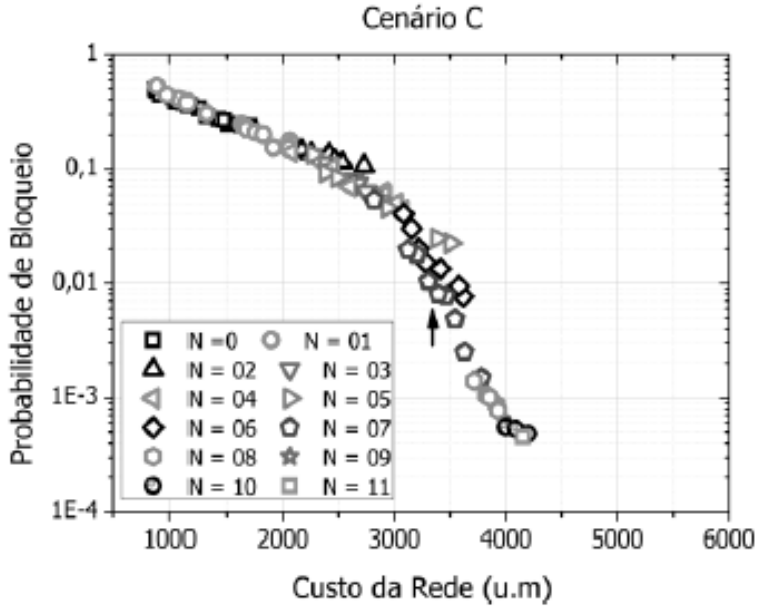

Figura 3 - Pareto do cenário $\mathrm{C}$.

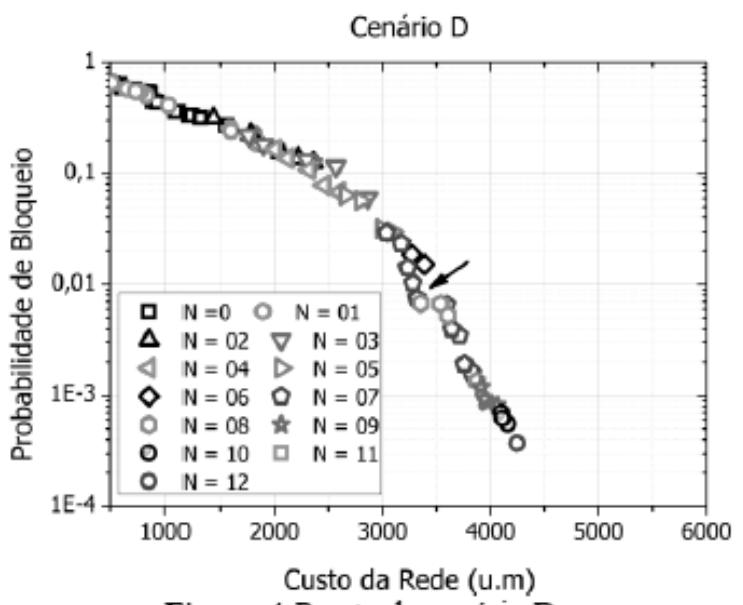

Figura 4-Pareto do cenário D.

Nos gráficos dos cenários $\mathrm{B}$ e $\mathrm{D}$ foi atingido a probabilidade de bloqueio de $1 \%$ com no mínimo 7 nós translucido e no cenário $\mathrm{C}$ foi atingido esse valor com 6 nós translúcidos. Mas como as soluções com 7 nós translúcidos são maioria nesse valor de probabilidade de bloqueio, elas foram utilizadas para analise mais detalhada. As redes da figura 5, 6 e 7 são configurações obtidas pelo MORWP simulados no cenário $\mathrm{B}, \mathrm{C}$ e D.

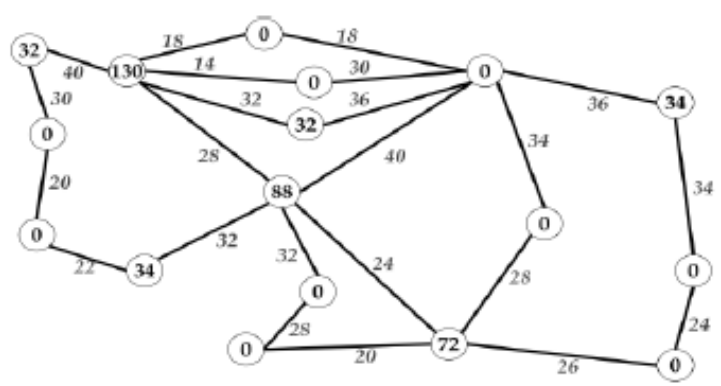

Figura 5 - Solução do algoritmo MORWP para o cenário $\mathrm{B}$. 


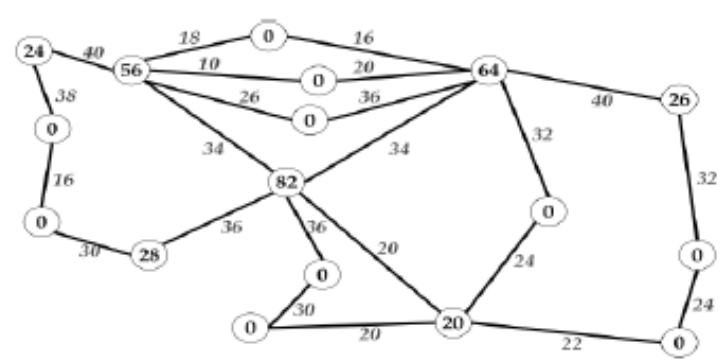

Figura 6 - Solução do algoritmo MORWP para o cenário $\mathrm{C}$

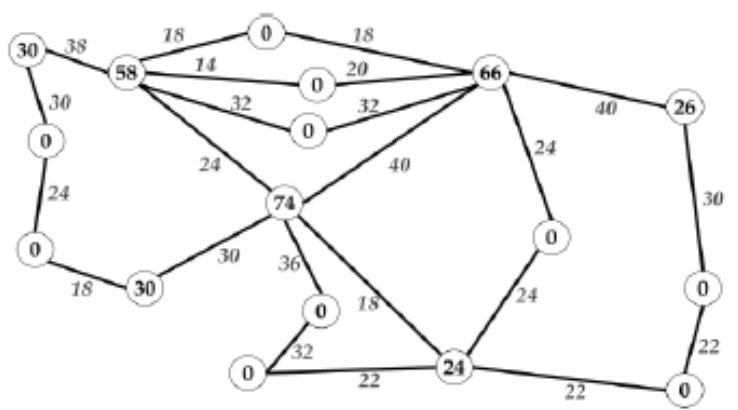

Figura 7 - Solução do algoritmo MORWP para o cenário D

Durante a interpretação dos resultados foi observado uma disparidade entre comprimentos de onda em enlaces que compartilham o mesmo nó em uma de suas extremidades. Primeiramente, foi inferido que isto se configurava como um erro do algoritmo, mas a partir de uma analise mais detalhada notou-se que o algoritmo privilegia os enlaces que possuem maior recorrência nas rotas durante as simulações. Foram feitos estudos de correlação entre a percentual de utilização do enlace em todas as rotas das simulações e a percentual de comprimento onda colocada nesse link em relação ao total colocado na rede. Em todos os experimentos a correlação atingiu uma taxa maior que $82 \%$, corroborando a qualidade do algoritmo.

As características das soluções escolhidas estão mostradas na tabela 5 e a partir desses valores foram montadas as redes, onde foram executados os algoritmos de RP, NDF e SQP. Foram montados 4 redes, duas com 28 comprimentos de ondas em todos os enlaces e 7 nós translúcidos com 44 regeneradores onde em uma executamos o NDF e na outra o SQP. As duas restantes, foram montadas com 30 comprimentos de ondas em todos os enlaces e 7 nós translúcidos com 62 regeneradores cada, onde foi executado o NDF e o SQP. Com essas quatro redes montadas mais as redes obtidas pelo MORWP foram realizadas simulações com variação de carga de 80 à 160 erlangs. O resultado está na figura 8 . Nessas simulações o MORWP se mostrou mais eficaz que o NDF e o SQP para todos os pontos de carga simulados.

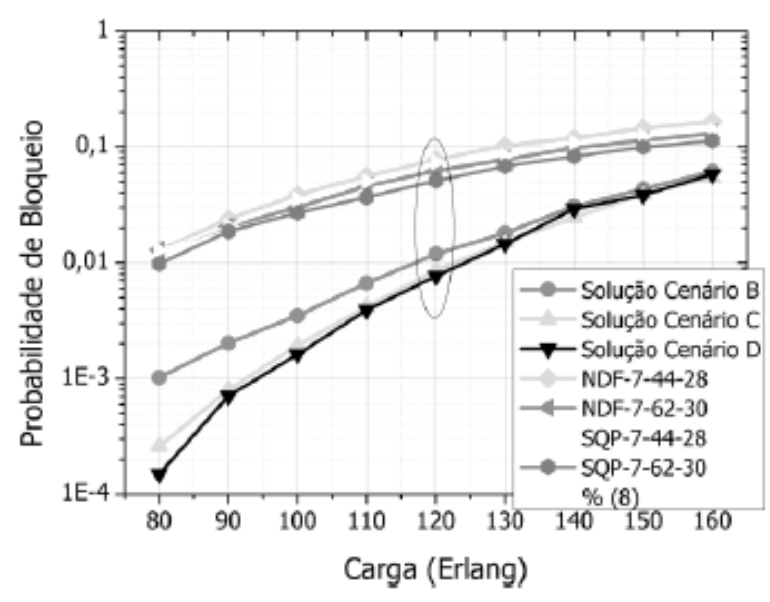

Figura 8 - Probabilidade de bloqueio em função da carga para as soluções exibidas anteriormente.

\section{Conclusões}

Nesse artigo foi proposto o uso de um algoritmo de otimização multiobjetiva evolucionária para solucionar o problema de posicionamento de regeneradores e comprimentos de onda, que chamamos de MORWP. O MORWP foi comparado com dois algoritmos de posicionamento de regeneradores presentes na literatura, NDF e SQP. Para um mesmo número de recursos na rede, o MORWP apresentou soluções com probabilidade de bloqueio menor que as soluções dos algoritmos NDF e SQP.

Nas simulações de carga o MORWP chega a alcançar uma probabilidade de bloqueio uma ordem de grandeza menor que o NDF e SQP no ponto de carga onde ocorreu seu treinamento. Os resultados mostram também que a estratégia de posicionar quantidades variáveis de regeneradores e comprimentos de ondas é melhor que posicionar blocos de quantidades fixas para ambos, como é feito no SQP e NDF.

A única ressalva a ser feita nesse comparativo é que o algoritmo proposto necessita de muito mais tempo de execução para resolver o problema, enquanto os outros dois são bem menos custosos em termos de desempenho. Mas isso não é nenhum empecilho já que esse algoritmos são executados durante a fase de planejamento da rede e não durante a operação da mesma.

\section{Referências}

[1] R. Ramaswami e K. N. Sivarajan, Optical Networks: A Practical Perspective, $2^{\mathrm{a}}$ ed. San Diego: Morgan Kaufmann, 2002. 
[2] B. Mukherjee, "WDM optical communication networks: Progress and challenges," Journal of Selected Areas in Communications, vol. 18, n. 10, p. 1810-1824, 2000.

[3] X. Yang e B. Ramamurthy, "Sparse regeneration in translucent wavelength-routed optical networks: Architecture, network design and wavelength routing," Photonic Network Communications, vol. 10, n. 1, p. 39-53, 2005.

[4] B. Ramamurthy et al, "Transparent vs. opaque vs. translucent wavelength-routed optical networks," OFC/IOOC 1999, vol. 1, p. 59-61, 1999.

[5] G. Shen e R. S. Tucker, "Translucent optical networks: the way forward," IEEE Communications Magazine, vol. 45, n. 2, p. 48-54, 2007.

[6] J.J. Grefenstette, “Optimization of Control Parameters for Genetic Algorithms," IEEE Trans. Systems, Man, and Cybernetics, Vol.SMC-16, No. 1, Jan./Feb. 1986, pp. 122-128.

[7] D. A. R. Chaves, C. F. C. L. C. Ayres, R. V. B. Carvalho, H. A. Pereira, C. J. A. Bastos-Filho e J. F. Martins-Filho. "Sparse Regeneration Placement for Translucent Optical Networks Using Multiobjective Evolutionary Algorithms Considering Quality of Service and Capital Cost", IMOC 2009, vol. 1. p. 417-422. 2009.

[8] D. A. R. Chaves, C. F. C. L. C. Ayres, R. V. B. Carvalho, H. A. Pereira, C. J. A. Bastos-Filho e J. F. Martins-Filho. "Multiobjective Sparse Regeneration Placement Algorithm for Optical Networks Considering Network Performance and Capital Cost," ICTON 2010. vol. 1. p. 1-4.

[9] D. A. R. Chaves, J. F. Martins-Filho, e C. J. A. Bastos-Filho, "Ferramenta Computacional para Simulação de Redes Ópticas Transparentes," in MOMAG 2008, vol. 1, 2008, p. 908-913.

[10] H. A. Pereira, D. A. R. Chaves, C. J. A. BastosFilho, and J. F. Martins-Filho, "OSNR model to consider physical layer impairments in transparent optical networks," Photonic Network Communications, vol. 18, n. 2, pp. 137-149, 2009.

[11] E. Zitzler, M. Laumanns, and L. Thiele. SPEA2: Improving the strength pareto evolutionary algorithm for multiobjective optimization. In K. Giannakoglou, D.

[12] K. F. Man, K. S. Tang, and S. Kwong, Genetic Algorithms: Concepts and Applications, IEEE Transactions on Industrial Electronics, vol. 43, p. 519, 1996.
[13] E. Zitzler. Evolutionary Algorithms for Multiobjective Optimization: Methods and Applications. PhD thesis, ETH urich, Switzerland, 1999. 